\title{
Erratum to: Nonlinear Wave Equations
}

\section{Erratum to:}

T. Li and Y. Zhou, Nonlinear Wave Equations, Series in Contemporary Mathematics 2, https://doi.org/10.1007/978-3-662-55725-9

In the original version of the book, the old list of editorial board has been updated with the new list in the Frontmatter.

The updated online version of this book can be found at https://doi.org/10.1007/978-3-662-55725-9 